\title{
BMJ Open Barriers faced by healthcare professionals when managing falls in older people in Kuala Lumpur, Malaysia: a qualitative study
}

\author{
Annaletchumy Loganathan, ${ }^{1}$ Chirk Jenn Ng, ${ }^{1}$ Maw Pin Tan, ${ }^{2}$ Wah Yun Low ${ }^{3}$
}

To cite: Loganathan A, $\mathrm{Ng}$ CJ, Tan MP, et al. Barriers faced by healthcare professionals when managing falls in older people in Kuala Lumpur, Malaysia: a qualitative study. BMJ Open 2015;5:e008460. doi:10.1136/bmjopen-2015008460

- Prepublication history for this paper is available online. To view these files please visit the journal online (http://dx.doi.org/10.1136/ bmjopen-2015-008460).

Received 11 April 2015 Revised 26 August 2015 Accepted 2 September 2015

\section{CrossMark}

\footnotetext{
${ }^{1}$ Department of Primary Care Medicine, Faculty of Medicine, University of Malaya, Kuala Lumpur, Malaysia

${ }^{2}$ Department of Medicine, Faculty of Medicine, University of Malaya, Kuala Lumpur, Malaysia

${ }^{3}$ Faculty of Medicine, Dean's Office, University of Malaya, Kuala Lumpur, Malaysia
}

Correspondence to Annaletchumy Loganathan; an18na03@yahoo.com

\section{ABSTRACT}

Objective: To explore the barriers faced by healthcare professionals (HCPs) in managing falls among older people (aged above 60 years) who have a high risk of falling.

Research design: The study used a qualitative methodology, comprising 10 in-depth interviews and two focus group discussions. A semistructured topic guide was used to facilitate the interviews, which were audio recorded, transcribed verbatim and checked for accuracy. Data were analysed thematically using WeftQDA software.

Participants: 20 HCPs who managed falls in older people.

Setting: This study was conducted at the Primary Care Clinic in the University Malaya Medical Centre (UMMC), Malaysia.

Results: Four categories of barriers emerged-these were related to perceived barriers for older people, HCPs' barriers, lack of caregiver support and healthcare system barriers. HCPs perceived that older people normalised falls, felt stigmatised, were fatalistic, as well as in denial regarding falls-related advice. HCPs themselves trivialised falls and lacked the skills to manage falls. Rehabilitation was impeded by premature decisions to admit older people to nursing homes. Lastly, there was a lack of healthcare providers as well as a dearth of fall education and training on fall prevention for HCPs.

Conclusions: This study identified barriers that explain poor fall management in older people with a high risk of falls. The lack of structured fall prevention guidelines and insufficient training in fall management made HCPs unable to advise patients on how to prevent falls. The findings of this study warrant evidence-based structured fall prevention intervention targeted to patients as well as to HCPs.

\section{INTRODUCTION}

The prevalence rate of falls among older individuals in lower to middle income countries varies from $10.1 \%$ in China to $54 \%$ in India, with $46 \%$ of the individuals in India

\section{Strengths and limitations of this study}

- The strength of this study lies in the recruitment of healthcare professionals (HCPs) from different disciplines, which provides in-depth understanding of the barriers to managing falls from different perspectives.

- This study found that issues such as trivialisation of falls, lack of clinical skills in managing falls and lack of interprofessional communication, were important barriers to suboptimal fall management among HCPs.

- Only HCPs' perspectives were included in this study. Older people's views and perceptions towards falls and fall prevention should be captured in future studies.

requiring medical attention following an incident fall. ${ }^{1}$ Malaysia is a middle-income country facing a rapid increase in the number of older people in its population, ${ }^{2}$ and additional healthcare services are needed to meet the increasing demand. ${ }^{3}$ Alongside population ageing, the number of older individuals presenting with falls in these communities are also expected to increase exponentially. Forty-seven per cent of older Malaysians attending a primary care clinic at a teaching hospital reported falls, and $60 \%$ of these fallers experienced resultant injury that needed medical attention in the preceding 12 months. ${ }^{4}$ In a separate rural population, $27 \%$ of older individuals living in a rural community reported falls in the previous year with $67 \%$ of falls occurring indoors. ${ }^{5}$ Falls are associated with increased dependency and mortality. A 10-year follow-up study of 198 older individuals presenting with falls at the emergency department of a teaching hospital revealed one, three, 5 and 10-year mortality rates of $22 \%$, $33 \%, 49 \%$ and $80 \%$, respectively, with significant deterioration in physical function reported after 1 year. ${ }^{6}$ 
Managing falls in older people is challenging because of their multifactorial nature. Contributing factors include environmental hazards, home hazards, poor health and functional disabilities. ${ }^{78}$ Despite there being effective interventions for preventing falls among older people, such as multi-component exercises, home hazard modifications, medication reviews, and multifaceted fall intervention programmes, ${ }^{9}$ healthcare professionals (HCPs) may not be ready to manage them in the community, due to various challenges. ${ }^{10-13}$ In addition, many fallers do not seek help. ${ }^{4}$ In a qualitative study, primary care physicians who received an academic outreach education intervention reported the barriers as being factors related to the physicians themselves, older people, logistics and systems. ${ }^{10}$ In another study, HCPs in emergency departments with an outreach education intervention exposure reported that the barriers were patient's compliance, shortage of HCPs and lack of reimbursement for successful patient referral. ${ }^{13}$ A systematic review of qualitative studies from the perspectives of community-dwelling older people and HCPs revealed various challenges in managing falls among older people, including cost, access to the intervention, time, social and cultural acceptance of assistive devices, exercise, fatalism and identity. ${ }^{14}$

To date, many qualitative and quantitative studies have been conducted on barriers to managing falls after fall prevention interventions are introduced in the clinical setting. ${ }^{15-17}$ Research on factors associated with poor fall risk assessment and management has tended to focus on the impact of outreach education intervention, ${ }^{10} 13$ and implementation of fall prevention clinical practice guidelines, ${ }^{17}$ and has been conducted in high-income countries. In our study, we explored barriers from the perspectives of HCPs who had not yet received an academic outreach education intervention and were managing falls among older multiethnic people in Malaysia, a middle-income country.

Therefore, seeking HCPs' perspectives on barriers to managing falls among older people in Malaysia would help identify ways to overcome the barriers, and provide insights into developing an effective fall prevention intervention for an older multiethnic population and HCPs.

\section{RESEARCH DESIGN AND METHODS}

This is a qualitative study that involved semistructured in-depth interviews (IDIs) and focus group discussions (FGDs) to explore barriers towards fall management among HCPs. ${ }^{18}$ In-depth interviews were one-on-one interviews for HCPs who were unable to join the focus group because of time constraints and conflicting duties. FGDs were group discussions involving 6-11 people, which allowed individuals to consider their views in the context of others' views. This approach can generate high-quality data. ${ }^{19}$ In this study, falls among older people were defined as 'unexpected events of coming to rest on the ground, floor, or lower level'. ${ }^{20}$
This study was conducted at the University Malaya Medical Centre (UMMC), a teaching hospital in an urban location in Malaysia. The primary inclusion criterion for this study was being an HCP from UMMC with experience in managing falls among people aged 60 years and above. Purposive sampling was employed, and participants from various backgrounds (discipline, years of experiences) were recruited to achieve maximum variation.

The topic guide used was developed based on a conceptual framework (figure 1) and clinical expert opinions. The topic guide, summarised in box 1, was used for participants in the IDIs and FGDs. The interviews were carried out between July 2012 and April 2013. The participants consented to be audio recorded, and all the interviews were carried out by ANN. To avoid potential participant response bias, the researcher did not interview close acquaintances or colleagues. Interviews were closely observed, and detailed notes were taken by a note taker. The IDIs lasted 30-50 min, while the FGDs lasted 60-80 min. Data collection ceased when data saturation was achieved; in other words, when ANN, NCJ and LWY reached a consensus that no new categories or themes were emerging from the interviews. The interview audio recordings were transcribed verbatim by ANN. ANN is competent in English and Malay, hence managed transcribing in both languages. Each transcript was checked for accuracy before being analysed. Interview quotations were verbatim; 11 interviews were conducted in English and one was in both, the Malay and English languages. This interview with some conversations in Malay was retained, and the conversations were translated only after the analysis was completed.

WeftQDA software (V.1.0.1) was used for data management and analysis. Thematic analysis was employed to identify the main themes. ${ }^{21}$ ANN and NCJ independently coded two transcripts to determine the coding framework. Coding discrepancies were resolved by discussions among ANN, NCJ and LWY, until consensus was reached. The coding framework was used to code the remaining transcripts by ANN. The new nodes that emerged during coding were added to the list of codes on consultation with NCJ and LWY.

Subsequently, the list of codes was grouped into larger categories that corresponded to the themes emerging from the data. Researchers' views were constantly reflected and debated so that any potential biases, which they might carry with them due to their backgrounds, were identified and addressed, to improve the accuracy of the findings.

\section{RESULTS}

\section{Sociodemographic profile of participants}

Thematic saturation was reached on the 12th interview. Twenty HCPs participated in this study out of the 35 who were approached to participate. Twelve HCPs declined to participate citing reasons such as time 


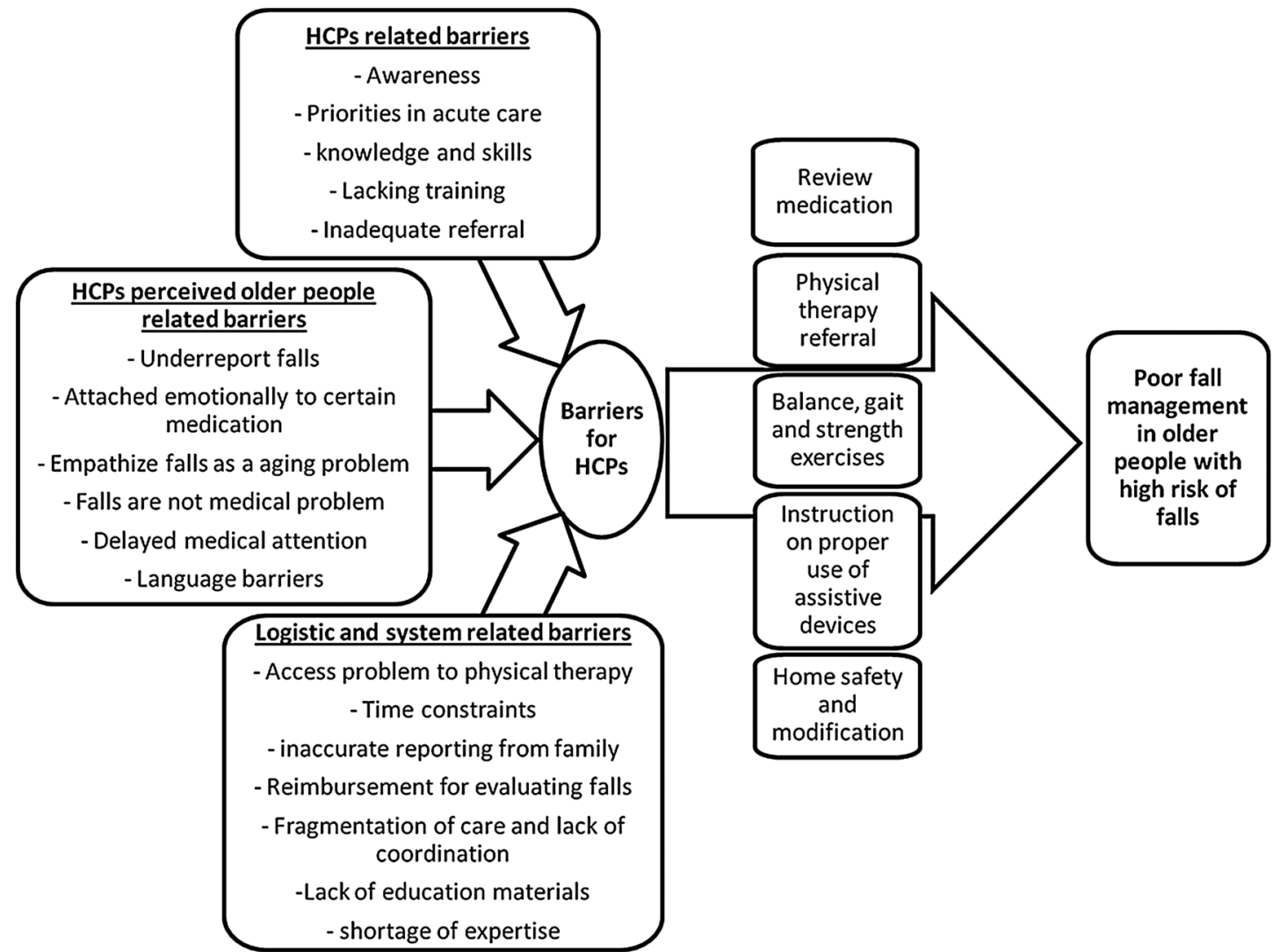

Figure 1 Conceptual framework for study. HCPs, healthcare professionals.

constraints, being on vacation, and not being interested in falls. The 20 participants recruited were from different specialties. The participants had a range of $1-15$ years of experience in managing falls. The participants' demographic details are shown in table 1. In total, 10 IDIs and two FGDs $(n=4 ; n=6)$ were conducted; the category participants in the FGDs included internal medicine $(n=4)$ and primary care $(n=6)$.

Box 1 Summary of the interview topic guide on barriers faced by healthcare professionals (HCPs) when managing falls in older people

Preamble: You have had experience in managing falls in older people with high risk of falls for some time. You would have advised and promoted evidence-based fall prevention strategies such as medication review, referral to physiotherapy, and home safety and modification, and prescribed assistive devices, such as a walking cane, to your patients.

- Can you share with me how you offer advice and manage your older patients' falls? (Probe: medication review, referral to physiotherapy, home safety and modification, and prescribed assistive devices) How?

Focusing on HCPs' barriers in managing falls

- What barriers do you face while managing falls in your older patients? (Probe: patient, doctors, healthcare system, caregivers)

- Do you face any problem when advising your patient on fall prevention strategies? (Probe: Access problem to physical therapy, vision and hearing, annoyed with home modification, exercise is not for fall prevention, too embarrassed to use a walking stick, the walking stick is for older people)

- Do you face any problems when managing falls in this hospital? (Probe: time, adequate HCPs, resources, facilities, transportation for patients)

- What barriers do you face when getting a fall history from an older patient? (Probe: language, denial, reporting falls, seek help for falls)

- There are some doctors who treat fall risk evaluation as not equivalent to cancer screening or congestive heart failure. Do you face this problem? How does it affect you when you manage falls in older people?

- Do your patients' families or caregivers take part when you offer advice on fall prevention? How do you find their support and care for fall problems for your patients?

- Do you face any problems while coordinating fall management with other HCPs in this hospital? (Probe: communication, role, coordination)

Do you think your patients face cost issues in addressing their falls? 
Table 1 Demographic details of HCPs

\begin{tabular}{lll}
\hline Characteristics & $\begin{array}{l}\text { Number } \\
(\mathbf{n = 2 0 )}\end{array}$ & Range \\
\hline Age (years) & & $29-54$ \\
Gender & & \\
$\quad$ Female & 14 & \\
$\quad$ Male & 6 & \\
Ethnicity & & \\
$\quad$ Malay & 7 & \\
$\quad$ Chinese & 7 & \\
Indian & 4 & \\
Other & 2 & \\
Professional background & & \\
$\quad$ Primary care doctors & 6 & \\
$\quad$ Internal medicine physicians & 4 & \\
$\quad$ Geriatricians & 4 & \\
Occupational therapist & 1 & \\
$\quad$ Physiotherapist & 1 & \\
Family medicine specialists & 2 & \\
$\quad$ Ophthalmologist & 1 & \\
$\quad$ Rehabilitation physician & 1 & \\
Experience in managing falls (years) & & $1-15$ \\
\hline HCPs, healthcare professionals. & &
\end{tabular}

\section{Emerging themes}

Four themes emerged from the analysis. These themes included perceived barriers for older people, HCP barriers, lack of caregiver support and healthcare system barriers for fall management. The main themes and category details are shown in table 2.

\section{Perceived barriers for older people}

Normalising falls

One HCP perceived that older people normalised falls and consider them to be part of the normal ageing process. He felt that older people disregarded falls because they believed they were inevitable in old age.

"It's because many of the older people do not see falls as a problem. In fact, some would say falls are normal as you get older. When you accept it as something normal you do not seek attention."

[Family Medicine Specialist (aged 36 years); 10 years' experience]

\section{Stigma around falls}

According to a HCP, older people viewed falling as a stigma because they associated falls with residential or nursing home admissions. Older people feared that their families might send them to nursing homes.

"The older person is unlikely to report their falls because as soon as they tell their families that they've fallen, they put them in a nursing home."

[Geriatrician (aged 38 years); 8 years' experience]

Table 2 Barriers faced by HCPs when managing falls among older people with a high risk of falls

Theme Categories

1. HCPs' perceived
barriers for older
people

\section{HCP barriers}
3. Lack of caregivers' support

\section{Healthcare system barriers}

1. Normalising falls

2. Stigma around falls

3. Reluctance to use assistive devices

4. Home modification issues

5. Fatalism

6. Denial

1. Trivialisation of falls

2. Lack of clinical skills in managing falls

3. Lack of inter-professional communication

1. Decision to admit to nursing home

2. Reinforcing older people's dependency

1. Lack of healthcare providers and facilities

2. Lack of continuity of care for fall management

3. Lack of fall education materials

4. Lack of HCP training on falls and their prevention

HCPs, healthcare professionals.

\section{Reluctance to use assistive devices}

Some older people considered using assistive devices, such as walking frames, as a stigma, because they believe that using such aids is a sign of weakness and reflects a change in one's self image.

\begin{abstract}
"We advise them to get a walking stick but they'd rather use an umbrella. Because they said that the walking stick doesn't look good on them."
\end{abstract}

[Family Medicine Specialist (aged 50 years); 12 years' experience]

\section{Home modification issues}

Issues such as inadequate space and living with a relative are challenges faced by occupational therapists when providing advice on home modification.

"Sometimes we from OT and PT side advise a patient 'ok, better to get a hospital bed'; but a patient instead says, 'I can get it, but in my house I don't have space for that bed."

[Occupational Therapist (aged 39 years); 6 years' experience]

Sometimes the house design itself is hazardous.

"I cannot remove that curb, you know village houses, right? The curb was built at the entrance of a house. If you remove that, then the rain water will enter the house." 
[Occupational Therapist (aged 39 years): 6 years' experience]

\section{Fatalism}

The HCP encountered obstacles with providing advice on fall prevention activities such as exercise and referral to physiotherapy. The older individuals may be fatalistic, whereby they believe that nothing more can be done to prevent their health from deteriorating or that death is around the corner.

"If I advise them (older people) to exercise or see a physiotherapist...they ask me instead, 'How could it help me to live longer, and why should I live longer? I'm dying soon. So why should I do exercise?"

[Physiotherapist (aged 33 years): 3 years' experience]

Furthermore, older people tended to adapt to living with deteriorating vision and refused to proceed with medical treatment to correct their vision.

"Usually, some patients will say 'I think it's ok, I'm old already, I don't need very good vision because I can still do my gardening, can still see my grandchildren's faces. I don't need to read a lot and do meticulous work and all that.' But they don't accept that in certain conditions they need good vision to see a slight amount of water on the floor; they can step on it, and then they fall because of it." [Ophthalmologist (aged 30 years); 1 year experience]

\section{Denial}

The HCP perceived that some older people refused to accept that they had a fall because of muscle weakness or knee, balance and gait problems.

"The older people sometimes do not want to admit their weakness or balance problem but they blame external factors like the environment, such as slippery floor, something blocked their vision and so on."

[Rehabilitation Physician (aged 41 years); 10 years' experience]

Older people also denied the risks of certain behaviour that could lead to falls.

"He was in hurry and he could not coordinate himself and fell again...I don't know (pause) they are careless when they want to be fast, they forget that they will fall down...It's not automatic anymore."

[Rehabilitation Physician (aged 41 years); 10 years' experience]

\section{HCPs' barriers}

Trivialisation of falls

Some HCPs 'under-assessed' the incidence of falls because they did not see it as a priority or as a presentation of something more serious. As a result, HCPs only provided basic consultations without exploring the possible causes of a fall.

"We sometimes don't think about the possible causes of the fall. We just think of it as another case of a fall and then go on to the next case."

[Internal Medicine Physician (aged 29 years); 2 years' experience]

\section{Lack of clinical skills in managing falls}

Many HCPs perceived that they lacked necessary knowledge and skills in managing falls. Some HCPs were unaware of the value of exercise in preventing falls, and that hearing problems can be a risk factor for falls. Other risks such as cataracts were underdiagnosed, and there was poor prescription of mobility aids for preventing falls.

"I would advise them not to exercise because I don't want them to fall again."

[Primary Care Physician (aged 31 years); 3 years' experience]

"I don't connect hearing impairment with fall, you know? I have not seen anyone fall because they are hearing-impaired."

[Geriatrician (aged 51 years); 13 years' experience]

"Cataract problems are under-detected for falls among older people."

[Primary Care Physician (aged 31 years); 5 years' experience]

\section{Lack of inter-professional communication}

Some HCPs felt that they had been working independently, without knowing relevant information and discussing it with other HCPs who were involved in fall management. Therefore, older people with falls were rarely referred across disciplines, such as to physiotherapists, pharmacists and occupational therapists.

"I think that there's not much communication in our team as well as the other teams to manage the patient although it should be multi-disciplinary, but actually we are working on our own and we don't really interact with each other." [Primary Care Physician (aged 34 years); 8 years' experience]

At times, the roles of physiotherapists and occupational therapists are poorly understood by some HCPs. As a result, some HCPs may not realise that some individuals who fall might benefit from rehabilitation, and hence they failed to prescribe these services.

"It is common that doctors here wrongly refer the patient to the physiotherapist for home visit ... that's why they 
need to know the difference between the physiotherapist and occupational therapist."

[Physiotherapist (aged 33 years); 3 years' experience]

\section{Lack of caregiver support}

Decision to admit to nursing homes

Some caregivers made a premature decision to admit their relatives suffering recurrent falls to nursing homes because they encountered difficulty in caring for them.

"Once a patient has one or two falls, they (family) will put him or her in a nursing home straight away because they are unable to take care of them at home (pause)." [Geriatrician (aged 38 years); 8 years' experience]

\section{Reinforcing older people's dependency}

Some caregivers were overly concerned about their older wards with recurrent falls and were hesitant to allow these people to walk independently. As a result, these older people became reliant on them for daily activities.

\begin{abstract}
"When you ask the family to supervise the older people, the family will come and hold his hand and they will not allow him to get down from the bed and walk from the chair without their help. So the older people becomes more dependent."
\end{abstract}

[Internal Medicine Physician (aged 30 years); 2 years' experience]

\section{Healthcare system barriers}

\section{Lack of healthcare providers and facilities}

The HCPs highlighted a shortage of personnel and time to manage falls among older people. Hence they were unable to screen older people for falls due to high patient loads and time constraints.

"I don't think we have sufficient amount of stuff and space to treat the rising number of elderly patients with fall. If I have 15 minutes to see one patient in the clinic and if I ask 'Did you have a fall recently?' I have to ask another minimum 10 more questions as to get the whole picture of patient's fall history, which is not practical in a clinic with a high patient load."

[Internal Medicine Physician (aged 35 years); 2 years' experience]

In addition, HCPs were not aware of services that sold adaptive tools for older people inside or outside the hospital. Therefore, they could not direct older people or caregivers to a point of purchase.

"The proper adaptive tools shop... (pause) I think that is the major problem. Because I was not informed of the availability of this shop and how to direct older people to go and get the tools?"

[Family Medicine Specialist (aged 36 years); 10 years' experience]

\section{Lack of continuity of care for fall management}

The lack of continuity of care was also an issue in fall management. The HCPs expressed that patients might see a different doctor for their next follow-up, hence this makes it difficult for them to manage these patients' falls, which should be monitored from baseline.

\section{"I might not see the patient in another visit, might be my colleague or MO (Medical Officer). So you would have told the patient something during this appointment, it might not be reinforced in the next appointment. That's it. And at the next appointment a different opinion may be given."}

[Rehabilitation Physician (aged 41 years); 10 years' experience]

There was also no follow-up for older people who were referred for home visits. After the first referral, the occupational therapist would visit the patient's home, and the necessary changes would be suggested. However, after that visit, the follow-up visits were never carried out.

"I don't know the effectiveness of educating patients on fall prevention during our home visit because we do it only one time, with no further follow-up after that." [Occupational Therapist (aged 35 years); 6 years' experience]

\section{Lack of fall education materials}

There was a lack of educational material about falls and fall prevention, and most HCPs who manage falls provided only verbal advice and drawings.

"We don't actually give patient any fall education material apart from just saying it. And the only thing we can do (pause) is referral for home visit when it's really the home environment that matters."

[Family Medicine Specialist (aged 50 years); 12 years of experience]

\section{Lack of HCP training on falls and fall prevention}

The HCPs felt that they received inadequate or no training on fall management. There was also a lack of discussions and conferences related to fall management.

"I think I was taught when I started working with the geriatrics consultant on how to deal with falls; only then I got it. It's not even emphasised in MRCP at all, and no one really emphasises the elderly as a special subset of patients or geriatrics as a special kind of subject on its own."

[Primary Care Physician (aged 34 years); 8 years' experience]

In addition, there was no clinical guideline available locally for fall management. 
“There's no structured programme. I think that we are unstructured in fall care so you tend to forget to ask something that might be important that might be the cause of the fall."

[Rehabilitation Physician (aged 41 years); 10 years' experience]

\section{DISCUSSION}

Many previous studies have explored barriers to effective implementation of a fall prevention intervention programme, ${ }^{10} 16172223$ especially in high income nations. This is the first study to highlight the barriers to managing falls among older people from the perspectives of HCPs in a lower to middle income country such as Malaysia. The barriers in fall management revealed by the HCPs were perceived barriers for patients, HCPs' barriers, lack of caregiver support and healthcare system barriers for fall management. Several themes were in common with previously published studies, such as lack of knowledge and skills in fall management, ${ }^{24}$ lack of training, ${ }^{16}$ poor interprofessional communication among HCPs, ${ }^{17}$ shortage of HCPs and family support, ${ }^{10}$ and older people underreporting falls, and refusing and not accepting walking aid usage. ${ }^{25} 26$

The HCPs expressed that older people perceived falls as normal and inevitable in old age. In another qualitative study, older people were found to normalise falls because they felt that falls were not medical; they felt that a fall is not a serious problem and that doctors are too busy to be bothered about patients' fall experiences. ${ }^{12}$ This may explain why there is poor reporting of falls by older people. Older people only report falls to doctors when the falls are serious. ${ }^{27} 28$ Studies have shown that Stepping On, a community-based fall prevention programme for older people, ${ }^{29}{ }^{30}$ and active health promotion in older individuals, ${ }^{31}$ can improve their knowledge as well as their behaviour about discussing their falls.

Stigma around falls and assistive devices is another obstacle for HCPs in managing falls. Older people feel embarrassed about falling and stigmatised because of their falls, and prefer to hide their perceived weakness as a result. ${ }^{28}$ This finding is common among older Chinese people. Older Chinese people in Hong Kong declined to use walking aids because they viewed them as a bad omen and a reflection of poor self-identity. ${ }^{32}$ In contrast, older people in Western culture, specifically Italian Canadians, were found to accept the use of aids when they really needed them or if their physicians prescribed their use. ${ }^{25}$ Perhaps there is an ethnic or cultural difference in terms of stigma related to the usage of walking aids. Hence, fall prevention messages should focus on positive health and social benefits, such as improving balance, and maintaining independence and social support from friends and family as facilitators to using a walking stick. ${ }^{33}$

Another barrier highlighted in this study is advice for older people on making significant interior home changes, which may not be possible due to inadequate space and home structure. In previous studies, many individuals viewed home modifications as unnecessary and intrusive, and they disliked changing loose rugs and attaching rails near toilets. ${ }^{34-36}$ Older women felt that a rail would interfere with the functional use of space in a small toilet area. Further, they may have rejected the idea of home modification not only to maintain their autonomy but also to maintain control over their home. ${ }^{34}$ However, they might accept such modifications if home visits or recommendations were to be mediated by their general practitioners. ${ }^{35}$ Therefore, HCPs may need to be the coordinators of care and to engage older people in making decisions about home hazard modifications.

In our study, fatalism was also an obstacle faced by HCPs in offering advice to older people on fall prevention. In one study, older Chinese people in England viewed falls as part of their destiny or punishment, and they were less likely to view falls as being preventable. ${ }^{28}$ Horne $e t a l^{27}$ reported fatalistic beliefs in older Caucasian as well as in South Asian people, including older Chinese people, who accepted falls as inevitable and an effect of ageing rather than a metaphysical consequence. These studies highlight, in different communities, social and cultural influences on fatalistic attitudes towards falling. ${ }^{14}$ Hence, HCPs need to take older people's views into account and to empathise with their beliefs and behaviour when offering advice on fall prevention strategies. ${ }^{35}$

HCPs also admitted that they themselves were barriers in managing falls by trivialising the fall circumstances and not recognising that there are balance exercises effective in preventing falls. In one study, HCPs were unaware that they should routinely address fall risk evaluation and management as they would other acute medical problems. ${ }^{10}$ Tinetti et $a l^{24}$ explained that HCPs encountered difficulties in assessing falls in older people who suffered from other chronic diseases. Greater priority was also usually given to managing chronic diseases such as coronary heart disease, cancer and diabetes, ${ }^{37}$ compared to managing falls. This could be due to the HCPs' lack of knowledge and skills to treat falls, while also managing their patients' comorbidities. ${ }^{13}$ In our study, HCPs expressed that, during their undergraduate and postgraduate studies, they lacked training to manage falls. ${ }^{3}$ Thus, the undergraduate and postgraduate curricula (medical, nursing and allied health professionals) need to be revised so that current and future generations of HCPs develop skills to assess and manage falls among older people.

HCPs also perceived poor support from caregivers as one of the barriers in effective fall management. For instance, some families make spontaneous decisions to admit a parent to a nursing home after the parent has had recurrent falls. However, this may be due to caregivers' work obligations and an inability to care for their parents at home. ${ }^{2}$ In this study, some caregivers were overly concerned about falls, reinforcing dependence in 
the older people and inadvertently increasing the risk of muscle weakness and frailty. Therefore, caregivers should be educated about the importance of fall prevention and they should facilitate reports about falls to physicians, so that recurrent falls and injuries can be prevented. ${ }^{10}$

HCPs indicated that the healthcare system in our setting lacks fall education materials such as booklets, pamphlets, posters, or videos to educate older people on fall prevention. To date, fall prevention education is delivered to older people verbally. In previous studies, the idea of giving out leaflets pertaining to fall prevention was seen as being intended as reinforcement for older people. ${ }^{30}$ However, older people have reported that HCPs' information and verbal advice is non-specific and impractical. ${ }^{26}$ Other than education materials, the healthcare system lacks follow-ups and continuity of care for older people who have had falls. Similar issues were raised by older Asian people who were not referred for any type of follow-up after attending an emergency department for fall-related injuries. ${ }^{12}$ Therefore, our setting needs structured fall education material, and all patients who have had falls and fall-related injuries need referral for fall prevention services, and should receive follow-ups as recommended by national and international bodies such as the National Institute for Health and Clinical Excellence, ${ }^{38}$ WHO, ${ }^{39}$ and the American and British Geriatrics Societies. ${ }^{40}$

\section{Strengths and limitations of the study}

The strength of this study lies in the recruitment of HCPs from different disciplines who are involved in the management of falls in older people. Thus, we were able to achieve in-depth understanding of the barriers to managing falls from various points of view. In addition, this study uncovered several barriers faced by HCPs while managing falls at the time when there were no structured guidelines for fall prevention in Malaysia. Hence, the findings of this study may be used to help in the development of structured guidelines for fall prevention in Malaysia and in other developing countries.

There are a few limitations in this study. Some disciplines, such as rehabilitation physician, occupational and physiotherapists, were represented only by a single participant. This may limit the transferability of the study. Furthermore, the group size for one of the FGDs was small $(n=4)$ and this may affect the group dynamics. Future studies should include participants from other states in Malaysia as well as from rural areas. In addition, only HCPs' perspectives were included in this study. However, this study is part of a larger study, and we are also exploring older people's views and perceptions towards falls and their prevention.

\section{Clinical recommendations}

HCPs perceived that older people faced access problems to fall prevention interventions. Therefore, HCPs should actively refer older people with a high risk of falls to attend a fall prevention intervention. Referral and education information about falls would enable older people to be aware of fall risks in the surrounding environment and to monitor their health condition. In addition, HCPs should continuously follow-up with referrals and reinforce them if follow-up is lacking. HCPs should also strive to recognise older people's social and cultural contexts before managing their fall problem. Lastly HCPs should discuss fall management and fall prevention, and create individualised plans that are mutually agreed on with older people and their families to facilitate fall prevention.

Dickson et $a l^{12}$ identified common challenges faced by older people (including Asian and Chinese populations) for fall prevention intervention as lack of referral, and behaviour, beliefs and context. Some of the challenges faced by older people may explain the barriers faced by HCPs in our study, such as lack of knowledge and training to: give referrals to educate older people about falls and their prevention; motivate older people to exercise; assess risk for falls; and to reinforce older people's efforts towards changing behaviours and beliefs about falls. Therefore, it is pertinent for the healthcare system to provide HCPs with the latest knowledge and training in fall prevention, so that they can empower older people to prevent falls and sustain their efforts effectively.

\section{Future recommendation}

This topic warrants more research to uncover other factors that could influence poor fall management among HCPs. In addition, exploring views from HCPs from other clinical settings, particular the north and east coasts of peninsular Malaysia and east Malaysia, is also vital and would provide comprehensive understanding about issues pertaining to poor fall management. In addition, exploring views from care providers and healthcare organisations would also provide insights about the barriers to HCPs cooperating to manage falls. This would allow triangulation of multiple sources of data, which may help to develop structured clinical guidelines for fall prevention and evidence-based fall prevention interventions in the local context.

\section{CONCLUSIONS}

This study identified the barriers in fall prevention and management from the perspectives of HCPs, and what they perceived to be patient barriers, HCP barriers, lack of caregiver support and healthcare system barriers. The lack of structured fall prevention guidelines and insufficient training on fall management led to HCPs' inability to effectively address fall prevention. However, ethnic and cultural differences among older people must be taken into account in tackling issues pertaining to fall prevention. The findings of this study indicate that structured evidence-based fall prevention interventions are warranted. 
Acknowledgements The authors would like to acknowledge Dr Lee Yew Kong, Ms Ranei Ho Gah Juan, Ms Syahidatul Akmal Azmi and Ms Tong Wen Ting, for their kind assistance. The authors also would like to thank the HCPs who participated in this research study, and the staff of the Department of Primary Care Medicine and Geriatrics Medicine, for their support.

Contributors This manuscript was drafted by AL, CJN, WYL and MPT. AL, CJN and WYL contributed to the qualitative study design and data analysis. AL was responsible for the grant application and ethical application for this project. All the authors contributed to the critical review of the manuscript and approved the final draft. All the authors read and approved the final version.

Funding The authors would like to thank the University of Malaya, Malaysia, for funding this project (IPPP-PG106-2012B: University of Malaya Postgraduate Fund). The authors also would like to thank the MyBrain15 (MyPhD) Scholarship Award from the Ministry of Higher Education, Government of Malaysia for the doctoral programme funding.

Competing interests None declared.

Ethics approval This study received ethical approval from the University Malaya Medical Centre (UMMC) Medical Ethics Committee (Reference: 926.2).

Provenance and peer review Not commissioned; externally peer reviewed.

Data sharing statement No additional data are available.

Open Access This is an Open Access article distributed in accordance with the Creative Commons Attribution Non Commercial (CC BY-NC 4.0) license, which permits others to distribute, remix, adapt, build upon this work noncommercially, and license their derivative works on different terms, provided the original work is properly cited and the use is non-commercial. See: http:// creativecommons.org/licenses/by-nc/4.0/

\section{REFERENCES}

1. Hua F, Yoshida S, Junling G, et al. Fall prevention in older age in Western Pacific Asia Region: WHO background paper to the global report on falls among older persons. Geneva, 2007.

2. Forsyth $\mathrm{D}$, Chia $\mathrm{Y}$. How should Malaysia respond to its ageing society. Med J Malaysia 2009;64:46-50.

3. Poi $P$, Forsyth $D$, Chan D. Services for older people in Malaysia: issues and challenges. Age Ageing 2004;33:444-6.

4. Sazlina S, Krishnan R, Shamsul A, et al. Prevalence of falls among older people attending a primary care clinic in Kuala Lumpur, Malaysia. J Community Health 2008;14:11-16.

5. Rizawati M, Mas Ayu S. Home environment and fall at home among the elderly in Masjid Tanah Province. J Health Transl Med 2008; $11: 72-82$

6. Tan M, Kamaruzzaman S, Zakaria M, et al. Ten-year mortality in older patients attending the emergency department after a fall. Geriatr Gerontol Int 2015 doi: 10.1111/ggi.12446. [Epub ahead of print].

7. Tinetti M, Baker D, McAvay G, et al. A multifactorial intervention to reduce the risk of falling among elderly people living in the community. N Engl J Med 1994;331:821-7.

8. Tinetti M, Doucette J, Claus E, et al. Risk factor for serious injury during falls by older persons in the community. J Am Geriatr Soc 1995;43:1214-21.

9. Gillespie L, Robertson M, Gillespie W, et al. Interventions for preventing falls in older people living in the community (Review). Cochrane Database Syst Rev 2012;(9):CD007146.

10. Chou W, Tinetti M, King M, et al. Perceptions of physicians on the barriers and facilitators to integrating fall risk evaluation and management into practice. J Gen Intern Med 2006:21:117-22.

11. Azidah A, Hasniza $\mathrm{H}$, Zunaina $\mathrm{E}$. Prevalence of falls and its associated factors among elderly diabetes in a tertiary center, Malaysia. Curr Gerontol Geriatr Res 2012;2012:539073.

12. Dickinson A, Horton K, Machen I, et al. The role of health professionals in promoting the uptake of fall prevention interventions: a qualitative study of older people's views. Age Ageing 2011;40: 724-30.

13. Fortinsky R, Lannuzzi-Sucich M, Baker D, et al. Fall-risk assessment and management in clinical practice: views from healthcare providers. J Am Geriatrics Soc 2004;52:1522-6.
14. Child S, Goodwin V, Garside R, et al. Factors influencing the implementation of fall-prevention programmes: a systematic review and synthesis of qualitative studies. Implication Sci 2012;7:1-14.

15. Häggqvist $B$, Stenvall M, Fjellman-Wiklund A, et al. "The balancing act"- Licensed practical nurse experiences of falls and fall prevention: a qualitative study. BMC Geriatr 2012;12:62.

16. Jones $\mathrm{T}$, Ghosh $\mathrm{T}$, Horn $\mathrm{K}$, et al. Primary care physician perceptions and practices regarding fall prevention in adult's 65 years and over. Accid Anal Prev 2011;43:1605-9.

17. Koh S, Manias E, Hutchinson A, et al. Nurses' perceived barriers to the implementation of a Fall Prevention Clinical Practice Guideline in Singapore hospitals. BMC Health Serv Res 2008;8:105.

18. Pope C, Mays N. Reaching the parts other methods cannot reach: an introduction to qualitative methods in health and health services research. BMJ 1995;311:42-5.

19. Merriam S. Qualitative Research: A Guide to Design and Implementation. USA: Jossey Bass, 2009.

20. Lamb SE, Jorstad-Stein EC, Hauer K, et al. Prevention of falls network Europe and outcomes consensus group. Development of a common outcome data set for fall injury prevention trials: the prevention of falls network Europe Consensus. J Am Geriatrics Soc 2005;53:1618-22.

21. Braun V, Clarke V. Using thematic analysis in psychology. Qual Res Psychol 2006;3:77-101.

22. Baker D, King M, Fortinsky F, et al. Dissemination of an evidence-based multicomponent fall risk assessment and management strategy throughout a geographic area. J Am Geriatrics Soc 2005;53:675-80.

23. Mackenzie L. Perceptions of health professionals about effective practice in falls prevention. Disabil Rehabil 2009;31:2005-12.

24. Tinetti M, Gordon C, Sogolow C, et al. Fall-risk evaluation and management: challenges in adopting geriatric care practices. Gerontologist 2006;46:717-25.

25. Aminzadeh F, Edwards N. Factors associated with cane use among community dwelling older adult. Public Health Nurs 2000;17:474-83.

26. Yardley L, Donovan-Hall M, Francis K, et al. Older people's views of advice about fall prevention: a qualitative study. Health Educ Res 2006;21:508-17.

27. Horne M, Speed S, Skelton D, et al. What do community-dwelling Caucasian and South Asian 60-70 year olds think about exercise for fall prevention? Age Ageing 2009;38:68-73.

28. Horton K, Dickinson A. The role of culture and diversity in the prevention of falls among older Chinese people. Can J Aging 2011;30:57-66.

29. Clemson L, Cumming R, Kendig $\mathrm{H}$, et al. The effectiveness of a community-based program for reducing the incidence of falls in the elderly: a randomized trial. J Am Geriatr Soc 2004;52:1487-94.

30. Clemson L, Swann M. Stepping on: Building confidence and reducing falls, a community based program for older people. Australia: Sydney University Press, 2008.

31. Dapp U, Anders J, von Renteln-Kruse W, et al. Active health promotion in old age: methodology of a preventive intervention programme provided by an interdisciplinary health advisory team for independent older people. J Public Health 2005;13:122-7.

32. Kong K, Lee F, Mackenzie A, et al. Psychosocial consequences of falling: the perspective of older Hong Kong Chinese who had experienced recent falls. J Adv Nurs 2002;37:234-42.

33. Stevens J, Noonan R, Rubenstein L. Older adult fall prevention: perceptions, beliefs, and Behaviors. Am J Lifestyle Med 2010;4:16-20

34. Clemson L, Cusick A, Fozzard C. Managing risk and exerting control: determining follow through with falls prevention. Disabil Rehabil 2015;21:531-41.

35. Simpson J, Darwin C, Marsh N. What are older people prepared to do to avoid falling? A qualitative study in London. $\mathrm{Br} J$ Community Nurs 2003;8:152, 4-9.

36. Krusea R, Moorea C, Toflea R, et al. Older adults' attitudes toward home modifications for fall prevention. $J$ Housing Elder 2010;24:110-29.

37. Sinnott C, Mc Hugh S, Browne J, et al. GPs' perspectives on the management of patients with multimorbidity: systematic review and synthesis of qualitative research. BMJ Open 2013;3:e003610.

38. Falls in older people: assessing risk and prevention. National Institute for Health and Clinical Excellence (NICE). London: NICE, 2013.

39. World Health Organization. Global Report on Falls Prevention in Older Age. France, 2007. http://www.who.int/violence_injury_ prevention/other injury/falls/en/

40. American Geriatrics Society, The Bristish Geriatrics Society. Clinical practice guidelines: prevention of falls in older persons. $J A m$ Geriatrics Soc 2010;59:148-1157. 